\title{
ON A THEOREM OF REPRESENTATION
}

\section{J. ABDELHAY}

Introduction. The main result of this paper is the characterization of the linear ring $(B C)$ of all bounded continuous real functions defined in a locally compact Hausdorff space which vanish at infinity (see Theorem 5). This is done by making use of some of the properties of the functional $\phi(x)=$ greatest lower bound of $x$, defined for the elements of $(B C)$ (see $\S .1$ and $\S .8$ ).

In this study we noticed that $\phi$ could give to the linear ring $(B C)$ both its structures of natural partial order and topology. We had then the idea of seeing when this could be done in general, that is to say, when is it true that for a partially ordered normed linear ring or a normed linear lattice there is a functional which could give both the structures of partial order and topology of the space. The rest of our paper deals with such a question.

For the demonstration of our theorem 5 we made very much use of Professor Stone's paper, A general theory of spectra, I, the reading of which we recommend for having a clear understanding of this one.

We are deeply indebted to Professors I. Kaplansky and M. H. Stone for many valuable suggestions and to Dr. L. Nachbin for kind discussions.

1. Definition of $S$-space. A linear space with real scalars $V$ is said to be an $S$-space with respect to a real function $\phi(x)$, defined in $V$, if and only if

(S1) $\phi(x) \leqq 0$ for any $x \in V$;

(S2) $\phi(x+y) \geqq \phi(x)+\phi(y)$ for any $x \in V$ and any $y \in V$;

(S3) $\phi(\lambda x)=\lambda \phi(x)$ for any $x \in V$ and any real number $\lambda \geqq 0$;

(S4) $\phi(0)=0$;

(S5) $\phi(x)+\phi(-x)=0$ implies $x=0(x \in V)$;

(S6) the equations in the unknown $\omega$

$$
\phi(\omega)=0, \quad \phi(\omega-x)=0
$$

have a solution $x^{+} \in V$ which is such that any other solution $x^{\prime} \in V$ of (E) satisfies the condition $\phi\left(x^{\prime}-x^{+}\right)=0$;

(S7) $\lim _{n, m \rightarrow \infty} \phi\left(x_{n}-x_{m}\right)=0$ implies the existence of an element $x \in V$ such that $\lim _{n \rightarrow \infty} \phi\left(x_{n}-x\right)=\lim _{n \rightarrow \infty} \phi\left(x-x_{n}\right)=0 \quad\left(\left\{x_{n}\right\}\right.$ is a sequence of elements of $V$ ).

Received by the editors March 4, 1948, and, in revised form, April 2, 1948. 


\section{Examples of $S$-spaces.}

ExAmple 1. The set $B$ of all bounded real functions defined in a topological space is an $S$-space with respect to the function

$$
I(x)=\text { g. 1. b. }\left(x_{-}\right) \quad(x \in B),
$$

where g.1.b. means "greatest lower bound of" and $x_{-}$is the negative part of $x$.

Proof. (1) $I(x) \leqq 0$ because $x_{-} \leqq 0$; (2) we have $x_{-}+y_{-} \leqq(x+y)_{-}$ and consequently $I(x)+I(y) \leqq$ g.1.b. $\left(x_{-}+y_{-}\right) \leqq I(x+y)$; (3) we have $(\lambda x)_{-}=\lambda x_{-}$for any real number $\lambda \geqq 0$ and so $I(\lambda x)=\lambda I(x)$ for $\lambda \geqq 0$; (4) $I(0)=0$ evidently; (5) we have $I(x)+I(-x) \leqq$ g.l.b. $\left(x_{-}+(-x)_{-}\right)$ =g.l.b. $(-|x|)$ and consequently $I(x)+I(-x)=0$ implies $x=0$; (6) we have $\left(x_{+}\right)_{-}=\left(x_{+}-x\right)_{-}=0$ where $x_{+}$is the positive part of $x$ and consequently $I\left(x_{+}\right)=0$ and $I\left(x_{+}-x\right)=0$. If $x^{\prime}$ is such that $I\left(x^{\prime}\right)=0$ and $I\left(x^{\prime}-x\right)=0$ then $x^{\prime} \geqq 0$ and $x^{\prime} \geqq x$. But now $x^{\prime} \geqq x_{+}$and consequently $I\left(x^{\prime}-x_{+}\right)=0$; (7) $I\left(x_{n}-x_{m}\right)+I\left(x_{m}-x_{n}\right) \leqq$ g.1.b. $\left(-\left|x_{n}-x_{m}\right|\right)$ and consequently $I\left(x_{n}-x_{m}\right) \rightarrow 0$ implies $\left(x_{n}-x_{m}\right) \rightarrow 0$ uniformly. But now there is an $x \in B$ such that $\left(x_{n}-x\right) \rightarrow 0$ uniformly. Consequently $\left(x_{n}-x\right)_{-} \rightarrow 0$ uniformly, that is to say, to each positive $\epsilon$ there is a suitable integer $N$ such that $-\epsilon<\left(x_{n}-x\right)_{-}<\epsilon$ for $n \geqq N$. Consequently

$$
-\epsilon \leqq I\left(x_{n}-x\right)<\epsilon \quad \text { for } n \geqq N \text {, }
$$

which means $\lim I\left(x_{n}-x\right)=0$. Similarly we get $\lim I\left(x-x_{n}\right)=0$.

ExAmple 2. The set $C$ of all continuous real functions defined in a compact Hausdorff space is an $S$-space with respect to the function $I(x)$ considered above (the proof of this is easily deducible from that of Example 1).

EXAMPLE 3. The set $L^{p}$ of all real functions defined in a compact measure space which have integrable $p$ th potence $(p \geqq 1)$ is an $S$-space with respect to the function

$$
J(x)=-\left(\int\left(-x_{-}\right)^{p}\right)^{1 / p} \quad\left(x \in L^{p}\right)
$$

Proof. (1) $J(x) \leqq 0$ evidently; (2) we have $-x_{-}-y_{-} \geqq-(x+y)_{-} \geqq 0$ and consequently $\left(\int\left(-x_{-}-y_{-}\right)^{p}\right)^{1 / p} \geqq\left(\int\left(-(x+y)_{-}\right)^{p}\right)^{1 / p}$. But we know from the theory of the integral that $\left(\int\left(-x_{-}\right)^{p}\right)^{1 / p}+\left(\int\left(-y_{-}\right)^{p}\right)^{1 / p}$ $\geqq\left(\int\left(-x_{-}-y_{-}\right)^{p}\right)^{1 / p}$ and consequently we have $-J(x)-J(y) \geqq$ $-J(x+y)$, that is to say $J(x+y) \geqq J(x)+J(y)$; (3) we have for $\lambda \geqq 0, \quad(\lambda x)_{-}=\lambda x_{-}$and consequently $J(\lambda x)=\lambda J(x)$ for $\lambda \geqq 0$; (4) $J(0)=0$ obviously; (5) $J(x)+J(-x)=0$ means in our case $J(x)=0$ and $J(-x)=0$ and consequently $x_{-}=0$ and $(-x)_{-}=0$. But then we 
have $x \geqq 0$ and $-x \geqq 0$ and therefore $x=0$; (6) see (6) of the proof of example 1 ; (7) we have $J\left(x_{n}-x_{m}\right)+J\left(x_{m}-x_{n}\right) \leqq-\left(\int\left(-\left(x_{n}-x_{m}\right)_{-}\right.\right.$ $\left.\left.-\left(x_{m}-x_{n}\right)_{-}\right)^{p}\right)^{1 / p}=-\left(\int\left|x_{n}-x_{m}\right|^{p}\right)^{1 / p} \leqq 0$. Therefore, $J\left(x_{n}-x_{m}\right) \rightarrow 0$ implies $\int\left|x_{n}-x_{m}\right|^{p} \rightarrow 0$. Consequently there is an $x \in L^{p}$ such that $\int\left|x_{n}-x\right|^{p} \rightarrow 0$. But $-\left(x_{n}-x\right)_{-} \leqq\left|x_{n}-x\right|$ and $-\left(x-x_{n}\right)_{-} \leqq\left|x_{n}-x\right|$. Consequently $J\left(x_{n}-x\right) \rightarrow 0$ and $J\left(x-x_{n}\right) \rightarrow 0$.

EXAmple 4. A Hilbert space is an $S$-space with respect to the function $H(x)=-\left\|x_{-}\right\|$, where $\|x\|$ is the norm of $x$.

EXAMPLE 5. The set of all bounded real functions defined in a topological space which vanish at some point of the topological space is an $S$-space with respect to the function

$$
I^{\prime}(x)=\text { g.l.b. }(x),
$$

where $x$ is a generic member of the set of functions.

\section{Structure of linear lattice for an $S$-space.}

Proposition 1. An $S$-space with respect to $\phi(x)$ is a linear lattice if we put: $x \geqq 0$ if and only if $\phi(x)=0$ and positive part of $x=x^{+}$ (see axiom (S6)).

Proof. (1) $x \geqq 0$ and $y \geqq 0$ imply $\phi(x+y)=0$ and consequently $x+y \geqq 0$; (2) $x \geqq 0$ and $\lambda \geqq 0$ imply $\phi(\lambda x)=0$ and hence $\lambda x \geqq 0$; (3) $x \geqq 0$ and $-x \geqq 0$ imply $\phi(x)=\phi(-x)=0$ and then $x=0$; (4) by (S6) we have $x^{+} \geqq 0$ and $x^{+} \geqq x$ and if $x^{\prime} \geqq 0$ and $x^{\prime} \geqq x$ then $x^{\prime} \geqq x^{+}$.

Proposition 2. In an $S$-space with respect to $\phi$ we have:

$$
x \geqq y \text { implies } \phi(x) \geqq \phi(y) \text {. }
$$

Indeed, we have $x=y+(x-y)$ and therefore $\phi(x) \geqq \phi(y)+\phi(x-y)$. Then, from the hypothesis $x \geqq y$ we get $\phi(x) \geqq \phi(y)$.

CoRollary. It is true that $\phi\left(x_{-}\right) \leqq \phi(x)$ and $\phi\left(x_{-}+y_{-}\right) \leqq \phi\left((x+y)_{-}\right)$.

\section{Norm for an $S$-space.}

Proposition 3. Given an $S$-space $L$ (with respect to $\phi$ ), if $g(r, s$ is any symmetric real function defined for $-\infty<r \leqq 0,-\infty<s \leqq 0$ such that

(D1) $g(r, s)>$ for $(r, s) \neq(0,0) ; g(0,0)=0$;

(D2) $g(\lambda r, \lambda s)=\lambda g(r, s)$ for any real number $\lambda \geqq 0$;

(D3) $g\left(r+r^{\prime}, s+s^{\prime}\right) \leqq g(r, s)+g\left(r^{\prime}, s^{\prime}\right)$;

(D4) $g(r, s)$ is monotone nonincreasing in each variable, then the real number $g(\phi(x), \phi(-x))$ is a norm for $L$ and we have

$$
0 \leqq x \leqq y \text { imply }\|x\| \leqq\|y\| \text {. }
$$


Proof. (1) For any $x \neq 0$ we cannot have simultaneously $\phi(x)$ $=\phi(-x)=0$ (by (S5)) and so, by (D1), $\|x\|>0$ for $x \neq 0$; for $x=0$ we have obviously $\|x\|=0$; (2) we have by (S3) and (D2): $g(\phi(\lambda x), \phi(-\lambda x))=\lambda g(\phi(x), \phi(-x))$ for $\lambda \geqq 0$ and consequently $g(\phi(-\lambda x), \phi(\lambda x))=-\lambda g(\phi(x), \phi(-x))$ for $\lambda<0$. But $g$ is symmetric, then $\|\lambda x\|=|\lambda|\|x\| ;(3)$ we have by (D4): $g(\phi(x+y), \phi(-x-y))$ $\leqq g(\phi(x)+\phi(y), \phi(-x)+\phi(-y))$. Then, by (D3), $\|x+y\| \leqq\|x\|+\|y\|$.

Let us prove now property $(\mathrm{N})$ : We have $\|x\|=g(\phi(x), \phi(-x))$ $=g(0, \phi(-x))$ for $x \geqq 0$ and similarly $\|y\|=g(0, \phi(-y))$ for $y \geqq 0$. If $x \leqq y$, then $\phi(-x) \geqq \phi(-y)$ and consequently $g(0, \phi(-x))$ $\leqq g(0, \phi(-y))$ (by (D4)), which means $\|x\| \leqq\|y\|$.

5. Indices for an $S$-space. A real function $\phi(x)$ defined in a linear space $L$ is said to be an index for $L$ if $L$ is an $S$-space with respect to $\phi$. Two indices of an $S$-space are said to be equivalent when they give rise to the same ordering and equivalent topologies.

An $S$-space $L$ with respect to an index $\phi$ satisfying the condition

$$
\phi\left(x_{n}\right) \rightarrow 0 \text { and } \phi\left(-x_{n}\right) \rightarrow 0 \text { imply } \phi\left(-\left|x_{n}\right|\right) \rightarrow 0
$$

is said to be a regular $S$-space.

Proposition 4. A regular $S$-space with respect to an index $\phi$ can be normalized in such a way that it becomes an S-space with respect to an index $W(x)$ equivalent to $\phi(x)$ and such that $W(x)=W\left(x_{-}\right)$.

Proof. The properties D1, 2, 4 of the function $g(r, s)$ considered above (see \$4) imply that $g(r, s) \rightarrow 0$ implies $(r, s) \rightarrow(0,0)$ and reciprocally $(r, s) \rightarrow(0,0)$ implies $g(r, s) \rightarrow 0$. We shall prove that the real function $W(x)=\phi\left(x_{-}\right)$is an index for our space and that $W$ is equivalent to $\phi$. In fact: (1) $W(x) \leqq 0$ obviously; (2) we have $W(x+y)$ $=\phi\left((x+y)_{-}\right) \geqq \phi\left(x_{-}+y_{-}\right)$because $(x+y)_{-} \geqq x_{-}+y_{-}$and consequently $W(x+y) \geqq W(x)+W(y)$; (3) $W(\lambda x)=\lambda W(x)$ for $\lambda \geqq 0$ obviously; (4) $W(0)=0$ evidently; (5) We have $W(x) \leqq \phi(x)$ and so $W(x)+W(-x)$ $=0$ implies $\phi(x)+\phi(-x)=0$, hence $x=0 ;$ (6) we have $W\left(x_{+}\right)$ $=W\left(x_{+}-x\right)=0$ obviously. If $x^{\prime}$ is such that $W\left(x^{\prime}\right)=W\left(x^{\prime}-x\right)=0$, then $x^{\prime} \geqq 0$ and $x^{\prime} \geqq x$ obviously. Then $x^{\prime} \geqq x_{+}$and consequently $W\left(x^{\prime}-x_{+}\right)=0$; (7) $W\left(x_{n}-x_{m}\right) \rightarrow 0$ means $\phi\left(\left(x_{n}-x_{m}\right)_{-}\right) \rightarrow 0$ and consequently $\phi\left(x_{n}-x_{m}\right) \rightarrow 0$. Then, by (S7), there is an $x$ such that $\phi\left(x_{n}-x\right) \rightarrow 0$ and $\phi\left(x-x_{n}\right) \rightarrow 0$. By (S8) we have then $\phi\left(-\left|x_{n}-x\right|\right) \rightarrow 0$. But $\left(x_{n}-x\right)_{-} \geqq-\left|x_{n}-x\right|$, then $W\left(x_{n}-x\right) \rightarrow 0$. Similarly we get $W\left(x-x_{n}\right) \rightarrow 0$.

Let us prove now that $W$ is equivalent to $\phi$. Indeed: (1) $W(x) \leqq \phi(x)$ and so $W(x)=0$ implies $\phi(x)=0$; reciprocally, $\phi(x)=0$ means $x \geqq 0$ and then $W(x)=0 ;(2)$ we have by (D4) 
$0 \leqq g(\phi(x), \phi(-x)) \leqq g(W(x), W(-x))=g\left(\phi\left(x_{-}\right), \phi\left((-x)_{-}\right)\right)$.

Then, when $g(W(x), W(-x)) \rightarrow 0$ we have that $g(\phi(x), \phi(-x)) \rightarrow 0$. Reciprocally, if $g(\phi(x), \phi(-x)) \rightarrow 0$, then, by our hypothesis, $\phi(x)$ and $\phi(-x)$ tend to zero. By (S8), $\phi(-|x|) \rightarrow 0$. But $\phi\left(x_{-}\right) \geqq \phi(-|x|)$ and $\phi\left((-x)_{-}\right) \geqq \phi(-|x|)$. Then $\phi\left(x_{-}\right) \rightarrow 0, \phi\left((-x)_{-}\right) \rightarrow 0$ and so $g(W(x), W(-x))$ tends to zero.

Finally, $W\left(x_{-}\right)=\phi\left(x_{-}\right)=W(x)$.

Proposition 5. For a regular S-space we have:

$$
\begin{aligned}
\left\|x_{-}\right\| & \leqq\left\|x_{\|}\right\|, \\
\|(x+y)-\| & \leqq\left\|x_{-}+y_{-}\right\| .
\end{aligned}
$$

Proof. (1) we have $\left\|x_{-}\right\|=g\left(W\left(x_{-}\right), 0\right)=g(W(x), 0) \leqq g(W(x)$, $W(-x))=\|x\| ;(2)$ we have $-(x+y)_{-} \leqq-\left(x_{-}+y_{-}\right)$, then, by $(\mathrm{N})$, $\left\|-(x+y)_{-}\right\| \leqq\left\|-\left(x_{-}+y_{-}\right)\right\|$and so $\left\|(x+y)_{-}\right\| \leqq\left\|x_{-}+y_{-}\right\|$.

6. Regular $S$-spaces and quasi Banach lattices. A quasi Banach lattice is a linear lattice which is also a Banach space with respect to a norm $\|\cdot\|$ satisfying conditions (N1) and (N) (see $\$ 4$ ).

THeOREM 1. A linear space $L$ is a quasi Banach lattice if and only if $L$ is a regular $S$-space.

Proof. We shall prove first that a regular $S$-space is a quasi Banach lattice. Indeed: (1) by Proposition 1, a regular $S$-space $L$ is a linear lattice; (2) by Proposition 3, $L$ is a normed space; (3) by Propositions 3 and 5, the norm satisfies conditions (N) and (N1). Then, all we have to do to prove that $L$ is a quasi Banach lattice is to show that $L$ is complete in the norm. Let $\left\{x_{n}\right\}(n=1,2, \cdots)$ be a sequence of $L$ and suppose $\left\|x_{n}-x_{m}\right\| \rightarrow 0$. This means (see $\$ 4$ ) that $g\left(\phi\left(x_{n}-x_{m}\right), \phi\left(x_{m}-x_{n}\right)\right) \rightarrow 0$. But now if we take $g$ such that $g(r, s)$ tends to zero implies $(r, s) \rightarrow(0,0)$ and reciprocally, we get: $\phi\left(x_{n}-x_{m}\right)$ tends to zero. By (S7) there is an $x \in L$ such that $\phi\left(x_{n}-x\right) \rightarrow 0$ and $\phi\left(x-x_{n}\right) \rightarrow 0$ and so $g\left(\phi\left(x_{n}-x\right), \phi\left(x-x_{n}\right)\right)=\left\|x_{n}-x\right\| \rightarrow 0$.

Let us prove now that a quasi Banach lattice is a regular $S$-space; precisely we shall prove that the function $\phi(x)=-\|x-\|$ is an index for the space and that this space is regular: (1) $\phi(x) \leqq 0$ obviously; (2) $\phi(x+y) \geqq \phi(x)+\phi(y)$ is easily deducible from (N2); (3) For $\lambda \geqq 0$, $\phi(\lambda x)=\lambda \phi(x)$ obviously; (4) $\phi(0)=0$ evidently; (5) $\phi(x)+\phi(-x)=0$ means in our case $x \geqq 0$ and $-x \geqq 0$ and consequently $x=0$; (6) see (6) of the proof of example $1, \S 1$; (7) we have $\phi\left(x_{n}-x_{m}\right)$ $+\phi\left(x_{m}-x_{n}\right)=-\left(\left\|\left(x_{n}-x_{m}\right)_{-}\right\|+\left\|\left(x_{m}-x_{n}\right)-\right\|\right) \leqq-\left\|\left|x_{n}-x_{m}\right|\right\|$. Consequently $\phi\left(x_{n}-x_{m}\right) \rightarrow 0$ implies $\left\|\left|x_{n}-x_{m}\right|\right\| \rightarrow 0$. But $\left(x_{n}-x_{m}\right)_{+}$ 
$\leqq\left|x_{n}-x_{m}\right|$ and $-\left(x_{n}-x_{m}\right)_{-} \leqq\left|x_{n}-x_{m}\right|$, hence, by $(\mathrm{N}),\left\|x_{n}-x_{m}\right\| \rightarrow 0$. Now there is an $x$ such that $\left\|x_{n}-x\right\| \rightarrow 0$. Then, by (N1), $\left\|\left(x_{n}-x\right)_{-}\right\| \rightarrow 0$, that is to say, $\phi\left(x_{n}-x\right) \rightarrow 0$. Similarly, we get $\phi\left(x-x_{n}\right) \rightarrow 0$.

Let us prove now that our $S$-space is regular. Let $\phi\left(x_{n}\right) \rightarrow 0$ and $\phi\left(-x_{n}\right) \rightarrow 0$. Then, $\left\|\left(x_{n}\right)_{-}\right\| \rightarrow 0$ and $\left\|\left(-x_{n}\right)_{-}\right\| \rightarrow 0$. Consequently, $\left\|\left|x_{n}\right|\right\| \rightarrow 0$. But -||$\left|x_{n}\right| \|=\phi\left(-\left|x_{n}\right|\right)$; then $\phi\left(-\left|x_{n}\right|\right) \rightarrow 0$.

Corollary. A Banach lattice, that is to say, a linear lattice which is also a Banach space with respect to a norm $\|\cdot\|$ such that

$$
|x| \leqq|y| \text { implies }\|x\| \leqq\|y\|,
$$

is a regular $S$-space.

Indeed, we have $\left|x_{-}\right| \leqq|x|$ and so $\left\|x_{-}\right\| \leqq\|x\|$, which is (N1), and $0 \leqq x \leqq y$ imply $\|x\| \leqq\|y\|$, which is $(\mathrm{N})$.

\section{Continuity of the operations in a regular $S$-space.}

Proposition 6. (a) $x \rightarrow x_{0}$ and $y \rightarrow y_{0}$ imply $(x+y) \rightarrow\left(x_{0}+y_{0}\right)$; (b) $x \rightarrow x_{0}$ implies $|x| \rightarrow\left|x_{0}\right|$.

PRoof. (a) we have $\left\|(x+y)-\left(x_{0}+y_{0}\right)\right\| \leqq\left\|x-x_{0}\right\|+\left\|y-y_{0}\right\|$, which proves part (a) of our proposition; (b) we have by (N1): $\left\|x_{-}\right\| \leqq\|x\|$ and $\left\|(-x)_{-}\right\| \leqq\|x\|$; consequently, $\||x|\| \leqq 2\|x\|$, which proves part (b) of our proposition.

Corollary 1. The operations $x \cup y$ and $x \cap y$ are continuous.

Indeed, we have $x \cup y=(x+y) / 2+|x-y| / 2$ and $x \cap y=(x+y) / 2$ $-|x-y| / 2$.

CoROllaRy 2. $x \leqq y, x \rightarrow x_{0}, y \rightarrow y_{0}$ imply $x_{0} \leqq y_{0}$.

Indeed, $x \leqq y$ means $x=x \cap y$ and consequently $x_{0}=x_{0} \cap y_{0}$ which says that $x_{0} \leqq y_{0}$.

Corollary 3. $x_{n} \leqq y_{n}, n=1,2, \cdots, \sum_{1}^{\infty} x_{n}=x, \sum_{1}^{\infty} y_{n}=y$ imply $x \leqq y$.

8. Definition of $S$-ring. A linear ring with real scalars $R$ is called an $S$-ring with respect to a real function $\phi(x)$, defined in $R$, if and only if:

(R1) $\phi(x)$ satisfies conditions (S1)-(S5) and (S7) (see \$1);

(R2) $\phi\left(x^{2}+2 \mu x\right)+\mu^{2} \geqq 0$ for $x \in R$ and any real number $\mu$;

(R3) $\phi(x)+\mu \geqq 0$ and $\phi(y)+\nu \geqq 0$ imply $\phi(x y+\nu x+\mu y)+\mu \nu \geqq 0$ for any real numbers $\mu$ and $\nu$ and $x \in R, y \in R$.

EXAmple 1. The set $B$ of all bounded real functions defined in a 
topological space is an $S$-ring with respect to the function $\phi(x)$ $=$ g.l.b. $\left(x_{-}\right)$.

Proof. We have already proved that $\phi$ satisfies (R1) (see example 1 of $\$ 1)$. Let us prove then that $\phi$ satisfies (R2) and (R3). In fact: $\left(x^{2}+2 \mu x\right)_{-}+\mu^{2}=\left(x^{2}+2 \mu x+\mu^{2}\right) \wedge \mu^{2} \geqq 0$, hence g.l.b. $\left(\left(x^{2}+2 \mu x\right)_{-}+\mu^{2}\right)$ $=$ g.l.b. $\left(x^{2}+2 \mu x\right)_{-}+\mu^{2} \geqq 0 ; \phi(x)+\mu \geqq 0$ gives $x_{-}+\mu \geqq 0$, hence $x+\mu$ $\geqq 0$ and $\mu \geqq 0$. Similarly we have $y+\nu \geqq 0$ and $\nu \geqq 0$. Then $x y+\nu x$ $+\mu y \geqq-\mu \nu$ and consequently $(x y+\nu x+\mu y)_{-} \geqq-\mu \nu$ because $-\mu \nu$ is negative.

Example 2. The set $C$ of all continuous real functions defined in a compact Hausdorff space is an $S$-ring with respect to the function $\phi(x)=$ g.l.b. $\left(x_{-}\right)$.

This is clear from the example 1.

EXAmPLE 3. The set $(B C)$ of all bounded continuous real functions defined in a locally compact Hausdorff space which vanish at infinity in an $S$-ring with respect to $\phi(x)=$ g.l.b. $(x)$.

PROof. It is clear that we have to prove only (R2) and (R3); (1) we have $x^{2}+2 \mu x+\mu^{2} \geqq 0$, hence g.l.b. $\left(x^{2}+2 \mu x\right)+\mu^{2} \geqq 0$; (2) $\phi(x)+\mu \geqq 0$ and $\phi(y)+\nu \geqq 0$ give $x+\mu \geqq 0$ and $y+\nu \geqq 0$, hence $x y+\nu x+\mu y+\mu \nu \geqq 0$ and then g.l.b. $(x y+\nu x+\mu y)+\mu \nu \geqq 0$.

Counter-eXample. The set $L$ is not an $S$-ring with respect to $\phi(x)=\int x \_d t$. Indeed, for $0 \leqq t \leqq 1, x=-t / 2, y=t, \mu=1 / 4, \nu=1 / 2$, condition (R3) is not satisfied, since $\phi(x)+\mu=0, \phi(y)+\nu=1 / 2$ and $\phi(x y+\nu x+\mu y)+\mu \nu=-1 / 24$.

\section{Partial order and norm for an $S$-ring.}

Proposition 7. In an S-ring with respect to $\phi(x)$ we have: (i) $\phi\left(x^{2}\right)=0$; (ii) $\phi(y)=0$ implies $\phi(x y-y \phi(x))=0$.

Proof. (i) is easily deducible from (R2) by putting $\mu=0$; (ii) is deducible from (R3) by putting $\mu=-\phi(x), \nu=0$.

Proposition 8. An S-ring with respect to $\phi(x)$ is a partially ordered normed linear ring by defining $x \geqq 0$ if and only if $\phi(x)=0$ and $\|x\|$ $=g(\phi(x), \phi(-x))$, where $g(r, s)$ is the function introduced in $\$ 4$.

This is clear from Propositions 1, 3 and 7.

Proposition 9. In an S-ring, with respect to $\phi$, which has a unit " $e$ " we have:

(E1) $\phi(-e) \geqq-1$;

(E2) $n x+e \geqq 0$ for any integer $n>0$ implies $x \geqq 0$;

(E3) $x-\phi(x) e \geqq 0$;

(E4) $\lambda e+x \geqq 0, \lambda e-x \geqq 0$ imply $\lambda \geqq \max (-\phi(x),-\phi(-x))$. 
Proof. (1) (E1) is clear from (R2) by making $x=e$ and $\mu=-1$; (2) we have $n x=-e+n x+e$, hence, by Proposition $2, \phi(n x)-\phi(-e)$ $\geqq \phi(n x+e)$. Since by hypothesis $\phi(n x+e)=0$ for any integer $n>0$, we get $\phi(n x) \geqq \phi(-e) \geqq-1$ and consequently $\phi(x) \geqq-1 / n$ for any $n>0$, which says that $\phi(x)=0$, hence $x \geqq 0$; (3) (E3) is clear from (ii) of Proposition 7 ; (4) we have $x+\lambda e-\lambda e=x$, hence, by Proposition $2, \phi(x+\lambda e) \leqq \phi(x)-\phi(-\lambda e)=\phi(x)-\lambda \phi(-e) \leqq \phi(x)+\lambda$; consequently, $\phi(x+\lambda e)=0$ implies $\phi(x)+\lambda \geqq 0$. Similarly we get $\phi(-x)+\lambda \geqq 0$.

\section{Sub $S$-ring of an $S$-ring.}

Proposition 10. If $R^{\prime}$ is an $S$-ring with respect to $\phi(x)$ which has no unit and if $R$ is the set of all pairs $(x, \mu)\left(x \in R^{\prime}, \mu\right.$ a real number $)$ with algebraic operations defined by $(x, \mu)+(y, \nu)=(x+y, \mu+\nu)$, $(x, \mu)(y, \nu)=(x y+\nu x+\mu y, \mu \nu), \lambda(x, \mu)=(\lambda x, \lambda \mu)$, then $R$ is an S-space with respect to $\psi(x, \mu)=\min (0, \phi(x)+\mu)$ and $R$ has a unit, precisely the element $(0,1)$.

The proof is trivial, and it is easily seen also that $R^{\prime}$ is a maximal ideal in $R$.

\section{1. $L$-space, $M$-space and ring of continuous functions.}

Theorem 2. An S-space $V$ with respect to a $\phi$ such that $\phi(x+y)$ $=\phi(x)+\phi(y)$ for $x \leqq 0, y \leqq 0$ is an L-space. ${ }^{1}$

Proof. We have seen that an $S$-space is a Banach space with norm $\|\cdot\|$ defined by $\|x\|=g(\phi(x), \phi(-x))$, where $g(r, s)$ is the function introduced in $\$ 4$, hence, in particular, $V$ is a Banach space with norm defined by $\|x\|=-\phi(x)-\phi(-x)$; (2) $V$ is a linear lattice (see Proposition 1); $x_{n} \leqq y_{n}, x_{n} \rightarrow x, y_{n} \rightarrow y$ imply $x \leqq y$ because $y-x \geqq y-x$ $+x_{n}-y_{n}$, hence $\phi(y-x) \geqq \phi\left(y-y_{n}\right)+\phi\left(x_{n}-x\right) \geqq-\left\|y-y_{n}\right\|-\left\|x_{n}-x\right\|$; finally, $x \geqq 0, y \geqq 0$ imply $\|x+y\|=-\phi(-x-y)=-\phi(-x)-\phi(-y)$ $=\|x\|+\|y\|$.

Theorem 3. An S-space with respect to a $\phi$ such that

$$
\phi(-x-y)=\min (\phi(x-y), \phi(y-x)) \text { for } x \cap y=0
$$

is an $M$-space. ${ }^{2}$

Proof. (1) Let $V$ be the $S$-space in question; then $V$ is a Banach

${ }^{1} \mathrm{~S}$. Kakutani, Concrete representation of abstract $(L)$-spaces and the mean ergodic theorem, Ann. of Math. vol. 42 (1941) pp. 523-537.

${ }^{2} \mathrm{~S}$. Kakutani, Concrete representation of abstract (M)-spaces, Ann. of Math. vol. 42 (1941) pp. 994-1024. 
space with norm defined by $\|x\|=\max (-\phi(x),-\phi(-x))$ and also a linear lattice; (2) $x_{n} \leqq y_{n}, x_{n} \rightarrow x, y_{n} \rightarrow y$ imply $x \leqq y$ because $\phi(y-x)$ $\geqq \phi\left(y-y_{n}\right)+\phi\left(x_{n}-x\right) \geqq-\left\|y-y_{n}\right\|-\left\|x_{n}-x\right\| ;$ (3) let $x \cap y=0$, then $\|x+y\|=-\phi(-x-y)=-\min (\phi(x-y), \phi(y-x))($ see $(M))=\|x-y\|$; (4) let $x \cap y=0$, then $-x-y \leqq-x \leqq-x+y,-x-y \leqq-y \leqq-y+x$, hence $\phi(-x-y) \leqq \phi(-x) \leqq \phi(y-x), \quad \phi(-x-y) \leqq \phi(-y) \leqq \phi(x-y)$. Consequently, $\phi(-x-y) \leqq \min (\phi(-x), \phi(-y)) \leqq \min (\phi(x-y)$, $\phi(y-x))$, hence $-\|x+y\| \leqq \min (-\|x\|, \quad-\|y\|) \leqq-\|x-y\|=$ $-\|x+y\|$. From this we get $\|x+y\|=\max (\|x\|,\|y\|)$.

Corollary. An S-space (with respect to $\phi) V$ which has an element 1 such that (i) $\phi(1)=0, \phi(-1)=-1$, (ii) $-\phi(x) \leqq 1$ implies $-x \leqq 1$ is an $M$-space and the element 1 is a Kakutani unit for the space $V$.

Proof. All we have to prove is that condition (M) holds in $V$. Indeed, we have $-x-y \leqq x-y,-x-y \leqq y-x$, hence $\phi(-x-y) \leqq \phi(x-y), \phi(-x-y) \leqq \phi(y-x)$ and consequently $\phi(-x-y)$ $\leqq \min (\phi(x-y), \phi(y-x))$. Let us prove now the inverse inequality. This is obvious if $k=\min (\phi(x-y), \phi(y-x))=0$, since in this case $x-y=0$ and therefore $x=y=0$ because $x \cap y=0$ by hypothesis. Suppose then $k<0$. As we have $\phi(x-y) \geqq k, \phi(y-x) \geqq k$, we get $-\phi((x-y) / k) \leqq 1,-\phi((y-x) / k) \leqq 1$, then, by hypothesis, $(x-y) / k$ $\leqq 1, \quad(y-x) / k \leqq 1$, hence $|x-y| \leqq|k| 1$. Consequently $\phi(-|x-y|)$ $\geqq \phi(-|k| 1)=|k| \phi(-1)=k$, which means, since $-(x+y)=-|x-y|$, that $\phi(-x-y) \geqq k$.

Finally we have: (1) $\|1\|=-\phi(-1)=1,1 \geqq 0$ because $\phi(1)=0$; (2) let $\|x\| \leqq 1$, that is to say, $\max (-\phi(x),-\phi(-x) \leqq 1$; then $-\phi(x) \leqq 1$, $-\phi(-x) \leqq 1$, hence $-x \leqq 1, x \leqq 1$, then $|x| \leqq 1$.

THEOREM 4. An S-ring with unit $R$ is a Stone normed ring ${ }^{4}$ and consequently a linear lattice. ${ }^{5}$

ProOF: (1) $R$ is a linear ring and is partially ordered (see Proposition $8)$; (2) if " $e$ " is the unit of $R$, then $n x+e \geqq 0$ for any integer $n>0$ implies $x \geqq 0$ (see Proposition 9, (E2)); (3) $\lambda e=x \geqq 0$ for some real number $\lambda$ (see Proposition 9, (E3)); (4) $R$ is complete in the norm

$$
\|x\|=\inf (\lambda ; \lambda x+e \geqq 0, \lambda x-e \geqq 0),
$$

which turns out to be $\|x\|=\max (-\phi(x),-\phi(-x)$ ) (see Proposition 9, (E4)).

${ }^{3}$ S. Kakutani, Ibid. p. 997.

${ }^{4}$ M. H. Stone, A general theory of spectra, I, Proc. Nat. Acad. Sci. U.S.A. vol. 26(1940) pp. 280-283.

5 M. H. Stone, Ibid. pp. 280-281. 
Corollary. An S-ring $R^{\prime}$ without unit is a linear lattice.

In fact, by Proposition $10, R^{\prime}$ is a maximal ideal of the ring $R$ defined in $\$ 10$ (see Proposition 10) and so, by Theorem 4 and Stone's result, ${ }^{6} R^{\prime}$ is a linear lattice, precisely a sublinear lattice of $R$.

Theorem 5. An S-ring without unit $R^{\prime}$ whose index $\phi$ is such that $\phi(x)=\phi\left(x_{-}\right)$is equivalent (=isometric, ring and lattice isomorphic) to the set $(B C)$ of all bounded continuous real functions defined in a locally compact Hausdorff space which vanish at infinity, and $\phi(x)=\inf \left(f_{x}(I)\right)$, where inf means greatest lower bound of and $f_{x}$ is the correspondent function of the element $x \in R^{\prime}$.

Proof. (1) $R^{\prime}$ is a maximal ideal of the $S$-ring $R$ defined in $\$ 10$ (see Proposition 10). This $S$-ring $R$ is a Stone normed ring (see Theorem 4) and so equivalent to the set $C$ of all continuous real functions defined in a compact space. ${ }^{7}$ This compact space is the set $M$ of all maximal ideals in $R$ (with an appropriate topology) and the correspondence between $R$ and $C$ is that one which associates to each $w \in R$ the real function $f_{w}(I)$, defined in the space $M$, such that $f_{w}(I) e-w \in I$ ( $e$ is the unit of $\left.R\right) .^{8}$ Then, if $w \in R^{\prime}$ we have $f_{w}\left(R^{\prime}\right) e-w$ $\in R^{\prime}$, hence $f_{w}\left(R^{\prime}\right)=0$, that is to say, if $f_{x}$ is the correspondent function of an element $x$ of $R^{\prime}$ then $f_{x}\left(R^{\prime}\right)=0$. We have, therefore, that $R^{\prime}$ is equivalent to the subset $(B C)$ of $C$ formed by all functions of $C$ which vanish in $R^{\prime} \in M$. Let us take off $R^{\prime}$ from $M$ : we get a new topological space $M^{\prime}$ which is locally compact and has $R^{\prime}$ as the point at infinity. We have also that now $(B C)$ is the class of all bounded continuous real functions defined in $M^{\prime}$ and which vanish at infinity; (2) let us prove now that $\phi(x)=$ g.l.b. $\left(f_{x}(I)\right)$ : we have $\|x\|=\sup _{I \in M}$ $\left|f_{x}(I)\right|, f_{x}$ being the correspondent function of $x \in R$. Suppose now that $x$ belongs to $R^{\prime}$, then $\|x\|=\max (-\phi(x),-\phi(-x))$ and so $\max (-\phi(x),-\phi(-x))=\sup _{I \in M}\left|f_{x}(I)\right|$. If $-x \geqq 0$, then $f_{-x}=-f_{x} \geqq 0$, $\phi(-x)=0$ and consequently $-\phi(x)=\sup _{I \in M}\left(-f_{x}(I)\right)$, that is to say $\phi(x)=\inf _{I \in M}\left(f_{x}(I)\right)$. For any $x \in R^{\prime}$ we have, by hypothesis, $\phi(x)$ $=\phi\left(x_{-}\right)$and so $\phi(x)=\inf _{I \in M}\left(f_{x_{-}}(I)\right)$. But $f_{x_{-}}=\left(f_{x}\right)_{-}$, so $\phi(x)$ $=\inf _{I \in M}\left(\left(f_{x}(I)\right)_{-}\right)$. The function $f_{x}$ vanishes at least once (at infinity), hence inf $\left(\left(f_{x}\right)_{-}\right)=\inf \left(f_{x}\right)$ and therefore $\phi(x)=\inf _{I \in M}\left(f_{x}(I)\right)$. But inf $I \in M\left(f_{x}\right)=\inf _{I \in M^{\prime}}\left(f_{x}\right)$, and so $\phi(x)=\inf _{I \in M^{\prime}}\left(f_{x}(I)\right)$.

UNIVERSITY OF BRAZIL

${ }^{6} \mathrm{M} . \mathrm{H}$. Stone, Applications of the theory of Boolean rings to general topology, Trans. Amer. Math. Soc. vol. 41 (1937) pp. 321-364; in particular, Theorem 80.

${ }^{7}$ M. H. Stone, loc. cit. footnote 5.

8 M. H. Stone, loc. cit. footnote 6, Theorems 76 and 80 . 